\title{
The Role of Spared Calcarine Cortex and Lateral Occipital Cortex in the Responses of Human Hemianopes to Visual Motion
}

\author{
Antony B. Morland ${ }^{1}$, Sandra L $\hat{e}^{1}{ }^{*}$, Erin Carroll $^{1}$, Michael B. Hoffmann ${ }^{1}$, \\ and Alidz Pambakian ${ }^{2}$
}

\begin{abstract}
Some patients, who are rendered perimetrically blind in one hemifield by cortical lesions, nevertheless exhibit residual visual capacities within their field defects. The neural mechanism that mediates the residual visual responses has remained the topic of considerable debate. One explanation posits the subcortical visual pathways that bypass the primary visual cortex and innervate the extrastriate visual areas as the substrate that underlies the residual vision. The other explanation is that small islands of the primary visual cortex remain intact and provide the signals for residual vision. We have performed behavioral and functional magnetic resonance imaging experiments to investigate the validity of the two explanations of residual
\end{abstract}

\section{INTRODUCTION}

Some hemianopes, with lesions to the geniculostriate projection, have sensitivity to brief or moving visual stimuli presented in their scotomata (Stoerig \& Cowey, 1997; Blythe, Bromley, Kennard, \& Ruddock, 1986; Blythe, Kennard, \& Ruddock, 1987; Weiskrantz, Warrington, Sanders, \& Marshall, 1974; Weiskrantz, 1986; Barbur, Ruddock, \& Waterfield, 1980; Riddoch, 1917). In addition to preserved visual sensitivity, patients are frequently able to discriminate reliably the direction of motion of visual stimuli (Azzopardi \& Cowey, 2001; Morland, 1999; Morland et al., 1999; Benson, Guo, \& Blakemore, 1998; Sahraie et al., 1997; Stoerig \& Cowey, 1997; Weiskrantz, Barbur, \& Sahraie, 1995; Perenin, 1991; Blythe et al., 1986, 1987; Barbur et al., 1980; Barbur, Watson, Frackowiak, \& Zeki, 1993). How might these direct visual responses of hemianopes be mediated? The parallel visual pathways that bypass the geniculostriate projection could subserve residual visual capacities (Weiskrantz et al., 1974). Anatomical (Cowey \& Stoerig, 1989; Standage \& Benevento, 1983; Benevento \& Yoshida, 1981; Fries, 1981; Yukie \& Iwai, 1981) and physiological (Girard

\footnotetext{
${ }^{1}$ Royal Holloway University of London, ${ }^{2}$ Imperial College School of Medicine

*Current address: INSERM U455, Service of Neurology, CHU Purpan, F-31059 Toulouse Cedex 3, France.
}

vision. Our behavioral experiments indicated that of the seven hemianopes tested, two had the ability to discriminate the direction of a drifting grating. This residual visual response was shown with fMRI to be the result of spared islands of calcarine cortical activity in one of the hemianopes, whereas only lateral occipital activity was documented in the other patient. These results indicate that the underlying neural correlates of residual vision can vary between patients. Moreover, our study emphasizes the necessity of ruling out the presence of islands of preserved function and primary visual cortex before assigning residual visual capacities to the properties of visual pathways that bypass the primary visual cortex.

\& Bullier, 1989; Girard, Salin, \& Bullier, 1991a, 1991b, 1992a; Rodman, Gross, \& Albright, 1989) studies on nonhuman primates clearly demonstrated that projections bypassing V1 innervate extrastriate areas. Recent functional imaging studies have indicated that extrastriate visual areas are active in human hemianopes, which have damage to the striate cortex (Goebel, Muckli, Zanella, Singer, \& Stoerig, 2001; Baseler, Morland, \& Wandell, 1999; Stoerig, Kleinschmidt, \& Frahm, 1998; Zeki \& Ffytche, 1998; Barbur et al., 1993). Furthermore, it would appear that monkeys (Pasik \& Pasik, 1971) and patients (Stoerig, Faubert, Ptito, Diaconu, \& Ptito, 1996) with lesions to striate and extrastriate visual areas have reduced or absent direct visual responses. The extrastriate visual areas would appear, therefore, to play a crucial role in mediating direct visual responses in destriate humans and monkeys. Moreover, there is evidence that in monkeys the middle temporal area, MT+/V5, maintains robust activity and directional selectivity when V1 is ablated (Rodman et al., 1989) or reversibly inactivated (Girard, Salin, \& Bullier, 1992b; Rodman et al., 1989). The MT+/V5 complex has, therefore, been put forward as the most likely structure to mediate discrimination of motion direction in the absence of V1. However, this view has recently been brought into question by Collins, Lyon, and Kaas (2003), who performed very similar experiments to those by Rodman et al. (1989), but failed to record activity in MT+/V5 in the absence of V1. 
Although there appears to be an elegant parallel between the behavioral and cortical responses in humans and monkeys, it is only in the monkey that complete lesions can be made with respect to the modularity of visual areas. In the light of this, another answer to what might mediate residual visual capacities in human hemianopes has emerged. Fendrich, Wessinger, and Gazzaniga (1992, 2001) proposed that spared tissue in the primary visual cortex could mediate residual visual responses that are found in human hemianopes. Strong evidence for this proposal was gained through very precise behavioral measurements that indicated that a hemianope could have small spared islands of visual sensitivity in an otherwise insensitive hemifield (Wessinger, Fendrich, \& Gazzaniga, 1997; Fendrich et al., 1992). These spared regions of visual sensitivity would go unnoticed with conventional perimetry and specialized equipment is required to locate the spared areas of visual field. More recent studies by other groups have also suggested that spared islands of sensitivity exist in patients with field defects (Wust, Kasten, \& Sabel, 2002; Scharli, Harman, \& Hogben, 1999; Kasten, Wuest, \& Sabel, 1998). However, no islands were identified in the much-studied hemianope, GY, using similar methodology (Kentridge, Heywood, \& Weiskrantz, 1997). A PET and SPECT imaging study on patients with extensive bilateral cortical lesions has documented how circumscribed regions of intact visual field can be correlated with residual activity in area 17 (Celesia, Bushnell, Toleikis, \& Brigell, 1991). However, other functional imaging studies have provided no positive evidence for spared islands of the primary visual cortex when patients' scotomata were stimulated (Goebel et al., 2001; Baseler et al., 1999; Stoerig et al., 1998; Zeki \& Ffytche, 1998; Barbur et al., 1993).

In this study, we examine which of the two theories described above best explains residual visual capacities in hemianopia. The residual visual capacity we chose to investigate was discrimination of the direction of motion of a large, drifting, sinusoidal grating. We selected this task because detection and discrimination of motion appear to be robust findings in those previously tested hemianopes with demonstrable residual vision. We test the hypothesis that the ability of hemianopes to discriminate the direction of moving gratings is associated with the sparing of the lateral occipital cortex and activity within it. In addition, we also test whether spared tissue in the primary visual cortex may provide lateral occipital motion areas with input and thereby mediate visual responses in hemianopes. In order to address these issues, we have used both behavioral and functional imaging approaches. We assessed the ability of seven hemianopes to discriminate the direction of motion of a horizontally drifting grating. The same grating, contrasted with a static grating, was then presented in a block design during acquisition of $\mathrm{T} 2{ }^{*}$ weighted magnetic resonance images. Our results indi- cate that, in the two subjects who were able to discriminate direction of motion of stimuli presented to their scotomata, the lateral occipital cortex was intact and active during motion stimulation. Further functional imaging experiments revealed that in one of the two subjects, activation could be elicited in the calcarine cortex, which suggests that spared islands of V1 may also mediate the residual visual responses in some, but not all, hemianopes.

\section{RESULTS}

\section{Subjects}

We tested seven hemianopic patients (AA, RC, RL, PF, SB, GY, and RA) and one patient with a clearly spared region of visual field in an otherwise blind hemifield (JP). Visual loss was associated with various different cortical lesions. Four of the patients (AA, RC, RL, and $\mathrm{PF})$ suffered lesions that involve the lateral aspects of the occipital lobe, which is associated with motion processing in normal subjects. In addition, these patients had lesions either to the optic radiation or early visual areas proximal or within the calcarine cortex. The remaining four patients ( $\mathrm{SB}, \mathrm{GY}, \mathrm{JP}$, and $\mathrm{RA}$ ) have lesions to the medial aspects of the occipital lobe with sparing of the lateral occipital region and, to a varying extent, sparing of the occipital pole. The details of each patient's lesion and visual fields are given in Table 1 and in Figure 1 lesion reconstructions are also superimposed on template slices (Talairach \& Tournoux, 1988) that cover the cortical areas normally involved in visual processing. Three patients' (GY, JP, and RA) visual fields and structural MRI images documenting their lesions are given in Figure 2. These patients are presented in greater detail because they were the three in whom we found intact direction discrimination, while the other patients had neither the ability to detect nor discriminate the moving stimuli presented (see Table 2 and Behavioral Responses section).

The patient GY, who has been described in detail previously (Barbur et al., 1980), has a lesion to the medial aspect of the left occipital lobe (Figure 2A). The lesion spares the occipital pole, and there is therefore a preserved region of intact visual field that extends horizontally to no greater than $3^{\circ}$ into the right visual field (Barbur et al., 1980). In our Humphrey visual field measurements, therefore, only a partial central sparing is observed because the $0.5^{\circ}$ target presented at $3^{\circ}$ eccentricity was not wholly within the spared region (Figure 2D). The subject JP also suffered a lesion to the medial aspect of the left occipital lobe particularly the anterior and inferior aspects (Figure 2B). Spared calcarine tissue can be seen, however, and this is consistent with intact regions in her visual fields (Figure 2E). The lesion suffered by RA is again located on the medial surface of the occipital lobe and spares little of the 
Table 1. Patient Details

\begin{tabular}{lllllll}
\hline Patient & Age & Sex & \multicolumn{1}{c}{ Aetiology } & Visual Fields & Lesion & Duration \\
\hline AA & 42 & M & Right AVM & L hemianopia & R medial and lateral occipital & 12 years \\
RC & 37 & F & Infarct & L hemianopia & R medial and lateral occipital + parietal & 10 years \\
RL & 34 & M & Resection meningioma & L hemianopia & R medial and lateral occipital + parietal & 8 years \\
PF & 52 & F & Infarct & R hemianopia & L lateral occipital + parietal + optic radiation & 11 years \\
SB & 39 & M & Tumor & L hemianopia & R medial occipital + parietal & 6 years \\
GY & 44 & M & Trauma & R hemianopia & L medial occipital $+R$ parietal & 36 years \\
RA & 65 & M & Infarct & R hemianopia & L medial occipital & 4 years \\
JP & 33 & F & Infarct & R field defect & L medial occipital & 7 years \\
\hline
\end{tabular}

$\mathrm{L}$ and $\mathrm{R}$ denote left and right, respectively; $\mathrm{AVM}=$ arteriovenous malformation.

Note that JP has extensive regions of intact visual field in the right hemifield.

calcarine cortex, although some tissue does appear present at the posterior and inferior aspects of this sulcus (Figure 2C). Visual field measurements reveal that the patient has some partially spared vision for targets presented in the superior right quadrant at eccentricities beyond $21^{\circ}$ (Figure $2 \mathrm{~F}$ ). The three patients, therefore, have lesions in very similar locations, but the area of spared cortex varies with the least sparing in GY and most in JP, who has also been shown to have a corresponding larger sparing of the visual field. Because of JP's intact visual field, we tested her in order to establish whether we could detect activity in the damaged hemisphere of a patient with a known region of visual sensitivity within a hemifield that was otherwise scotomatous.

We also tested five control subjects, who were aged between 21 and 61. Two subjects were men and three were women. The control subjects have normal vision and no other known neurological conditions.

\section{Behavioral Responses}

We presented horizontally drifting gratings $\left(10^{\circ} \times 10^{\circ}\right.$ centered on the horizontal meridian at an eccentricity

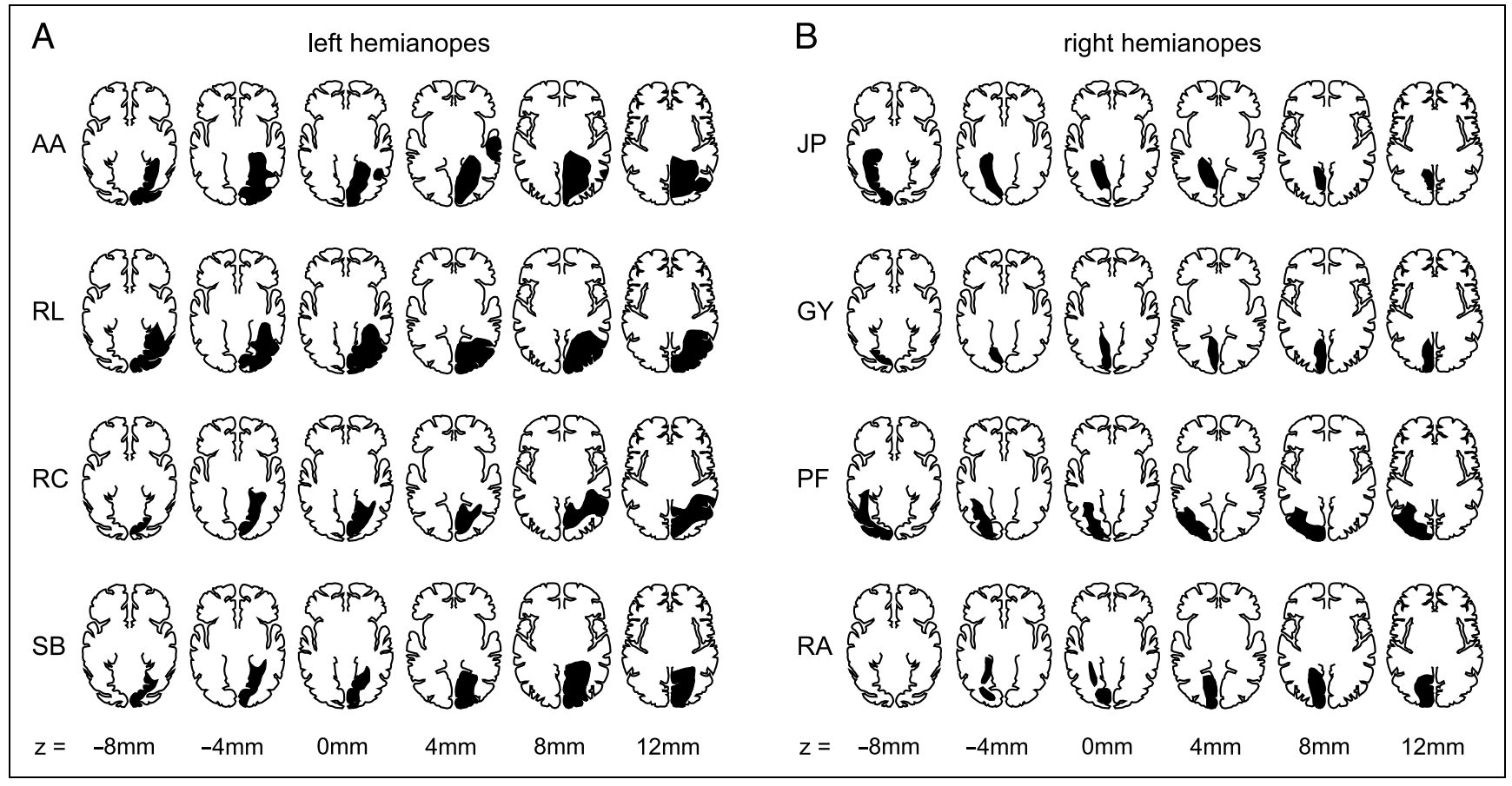

Figure 1. Lesion reconstructions for all patients. The filled areas indicate the site and extent of the lesions in each case, superimposed on slices extracted from the Talairach and Tournoux (1988) coordinate frame of reference. The slices shown are those that cover the calcarine cortex and the lateral occipital cortex. Many patients had tissue damage beyond the selected slices, the details of which may be found in Table 1 . 


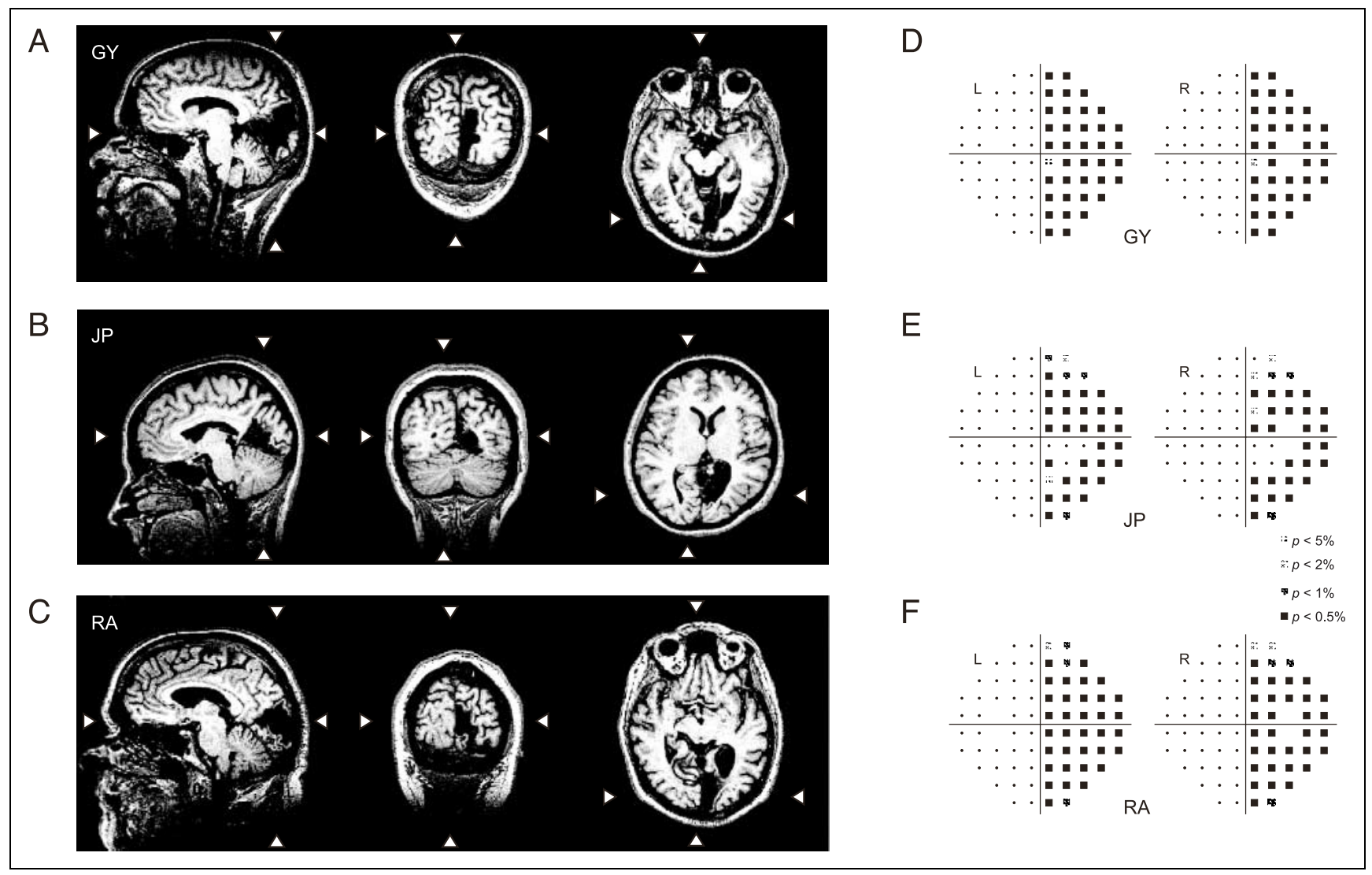

Figure 2. Anatomical T1-weighted images of three patients, GY (A), JP (B), and RA (C). On the left, center, and right of each panel, parasagittal coronal and axial slices are given in neurological convention. Slices were selected to best reveal the position and extent of the lesion. Arrowheads indicate the positions of the two slices perpendicular to the imaged slice in each panel. Humphrey visual field plots are given for GY (D), JP (E), and RA (F). Data were acquired with the 30-2 threshold protocol, which presented $0.5^{\circ}$ targets to the central $\pm 30^{\circ}$. The probability scores are presented for both eyes and indicate the homonymous nature of the defects. It should be noted that the solid squares indicate that the maximum instrument limited target luminance was undetected. Only in JP is there sparing of the visual field in areas where the visual stimulus was presented for behavioral and functional imaging experiments.

of $10^{\circ}$ ) to the subjects and asked them to indicate the direction of grating's motion. Within each block of trials we tested two speeds, $5 \% \mathrm{~s}$ and $10 \%$ and in separate blocks gratings of different spatial frequencies (0.5 and 1 cycle/deg) were presented. For all subjects, performance in the motion discrimination task was not influenced by either speed (test for independence $\chi^{2}$ range of $\left.0-2.38, d f=1, p>.10\right)$ or spatial frequency (test for independence $\chi^{2}$ range of $0.13-$ 1.19, $d f=1, p>.10)$. In Table 2 , therefore, the percentage of correct responses across conditions is given for each hemianope. All patients were able to perform the direction discrimination task, when stimuli were presented in their intact visual hemifields (Table 2), as were the normal control subjects (100\% in all cases). All patients with lateral occipital lesions (AA, RC, RL, and PF) and one with medial occipital lesions (SB) failed to respond better than predicted by chance when stimuli were presented in their scotomata (goodness-of-fit $\chi^{2}$ range of $0.0-3.1, d f=1$, $p>.05)$. Of the four patients with damage to medial aspects of the occipital lobe, three (GY, JP, and RA) were able to perform the task at levels greater than predicted by chance (goodness-of-fit $\chi^{2}$ range of 61.5-

Table 2. Behavioral Responses of Patients for Discrimination of a Drifting Sinusoidal Grating

\begin{tabular}{lcccccrrr}
\hline Subject & $A A$ & $R C$ & $R L$ & $P F$ & $S B$ & $G Y$ & $R A$ & $J P$ \\
\hline Intact & $100 \%$ & $100 \%$ & $100 \%$ & $100 \%$ & $93 \%$ & $100 \%$ & $100 \%$ & $100 \%$ \\
Scotoma & $53 \%$ & $57 \%$ & $55 \%$ & $51 \%$ & $50 \%$ & $87 \%$ & $100 \%$ & $98 \%$ \\
\hline
\end{tabular}

Figures are given as total percentage of correct responses. 
Table 3. Number of Voxels that Exceeded a Correlation of .23 $(p<.05)$ in the Lateral Aspects of the Occipital Lobe for Stimulation with a Drifting Grating

\begin{tabular}{|c|c|c|c|c|}
\hline \multirow[b]{3}{*}{ Subject } & \multicolumn{4}{|c|}{ Patients } \\
\hline & \multicolumn{2}{|c|}{$\begin{array}{l}\text { Stimulation of } \\
\text { Intact Hemifield }\end{array}$} & \multicolumn{2}{|c|}{$\begin{array}{l}\text { Stimulation } \\
\text { of Scotoma }\end{array}$} \\
\hline & Contralateral & Ipsilateral & Contralateral & Ipsilateral \\
\hline SB & 9 & 0 & 0 & 0 \\
\hline $\mathrm{RL}$ & 95 & 0 & 0 & 0 \\
\hline AA & 7 & 0 & 0 & 0 \\
\hline $\mathrm{PF}$ & 75 & 0 & 0 & 0 \\
\hline $\mathrm{RC}$ & 14 & 0 & 0 & 0 \\
\hline GY & 59 & 67 & 24 & 0 \\
\hline RA & 53 & 0 & 41 & 2 \\
\hline \multirow[t]{3}{*}{ JP } & 12 & 0 & 21 & 6 \\
\hline & \multicolumn{4}{|c|}{ Controls } \\
\hline & \multicolumn{2}{|c|}{$\begin{array}{l}\text { Stimulation of } \\
\text { Left Hemifield }\end{array}$} & \multicolumn{2}{|c|}{$\begin{array}{l}\text { Stimulation of } \\
\text { Right Hemifield }\end{array}$} \\
\hline Subject & Contralateral & Ipsilateral & Contralateral & Ipsilateral \\
\hline $\mathrm{MH}$ & 56 & 0 & 36 & 15 \\
\hline $\mathrm{EC}$ & 66 & 3 & 14 & 0 \\
\hline EA & 86 & 6 & 42 & 3 \\
\hline SM & 9 & 0 & 12 & 3 \\
\hline $\mathrm{MC}$ & 2 & 0 & 5 & 0 \\
\hline
\end{tabular}

80, $d f=1, p<.00001)$. All the patients who were capable of the discrimination task reported awareness of the stimulus, whereas the patients who were unable to perform the discrimination reported a complete absence of awareness of the stimulus. It should be noted, however, that we did not test awareness in a formal manner as others have (e.g., Weiskrantz et al., 1995). The visual stimulus presented to JP fell on regions of the visual field in which she maintained some sensitivity as determined by perimetry (Figure 2E), so her ability to discriminate stimulus motion direction is unsurprising.

We tested patients with lesions that included the lateral occipital cortex and those with lesions to the medial occipital cortex alone in order to test whether the presence of the lateral aspects of the occipital lobe was necessary for motion discrimination. Our behavioral measurements appear consistent with the notion that the lateral occipital areas are necessary (GY and RA) but not always sufficient for motion discrimination (SB).

\section{Cortical Responses}

We first wished to establish whether visual motion stimulation elicited cortical activation in lateral occipital areas in those subjects with intact motion discrimination. The second aim was to determine whether any spared tissue on the medial aspects of the occipital lobe may be activated by visual stimuli and hence contribute to the input to lateral occipital areas that may mediate motion discrimination. We met these goals by performing one imaging experiment in which the visual stimulus conditions were differentiated by motion, and another in which a grating undergoing contrast reversal was contrasted with uniform gray.

In all hemianopes and controls, cortical activation in lateral occipital locations was observed when moving stimuli were presented to intact regions of the visual field. In Table 3, the number of voxels that exceeded a correlation coefficient of .23 $(p<.05)$ is given for each subject. In the normal controls and in hemianopes stimulated in the intact hemifield, the activity was predominant in the hemisphere contralateral to the stimulation in all subjects apart from GY (Table 3). In GY similar numbers of voxels were active in the contralateral and ipsilateral hemispheres, a result that has been observed previously (Baseler, Wandell, Morland, Jones, \& Ruddock, 1997) and that is consistent with some behavioral responses to motion (Finlay, Jones, Morland, Ogilvie, \& Ruddock, 1997). The number of active voxels contralateral to the stimulated intact hemifield in the hemianopes was not significantly different from the number contralateral to the stimulated hemifield in the controls (Mann-Whitney $U$ test $=34, p=.59$ ). This

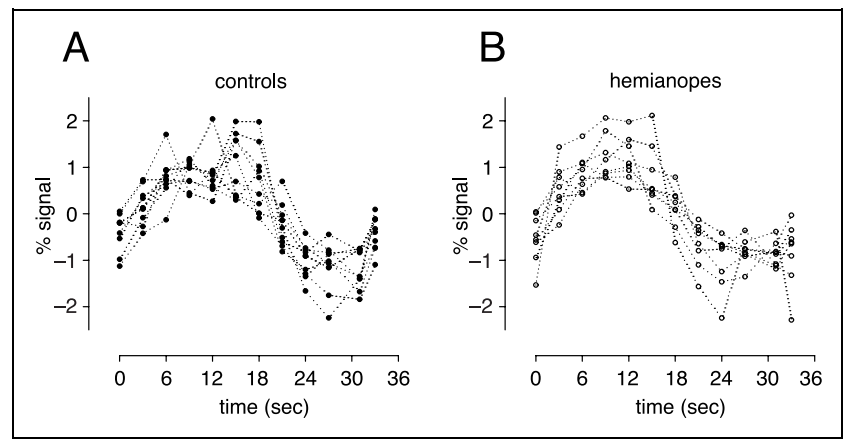

Figure 3. The mean BOLD signal expressed in terms of percentage change from the mean signal. The signals shown are the mean of six 36-sec periods of the stimulus cycle for two runs (apart from RC, where only one run was available). Data are given for each subject for regions of interest in the lateral occipital area for voxels that exceeded a correlation of $.23(p<.05)$. In A, data are given for 10 control hemispheres of the 5 control subjects tested. In $\mathrm{B}$, data are given for the intact hemisphere of each of the hemianopes. In each panel, the broad gray line indicates the overall mean of the group data. Note in each group that the response amplitude varies between approximately $\pm 0.5 \%$ and $\pm 2 \%$ and that the mean BOLD responses show the characteristic features associated with the low-pass nature of the hemodynamic response. 


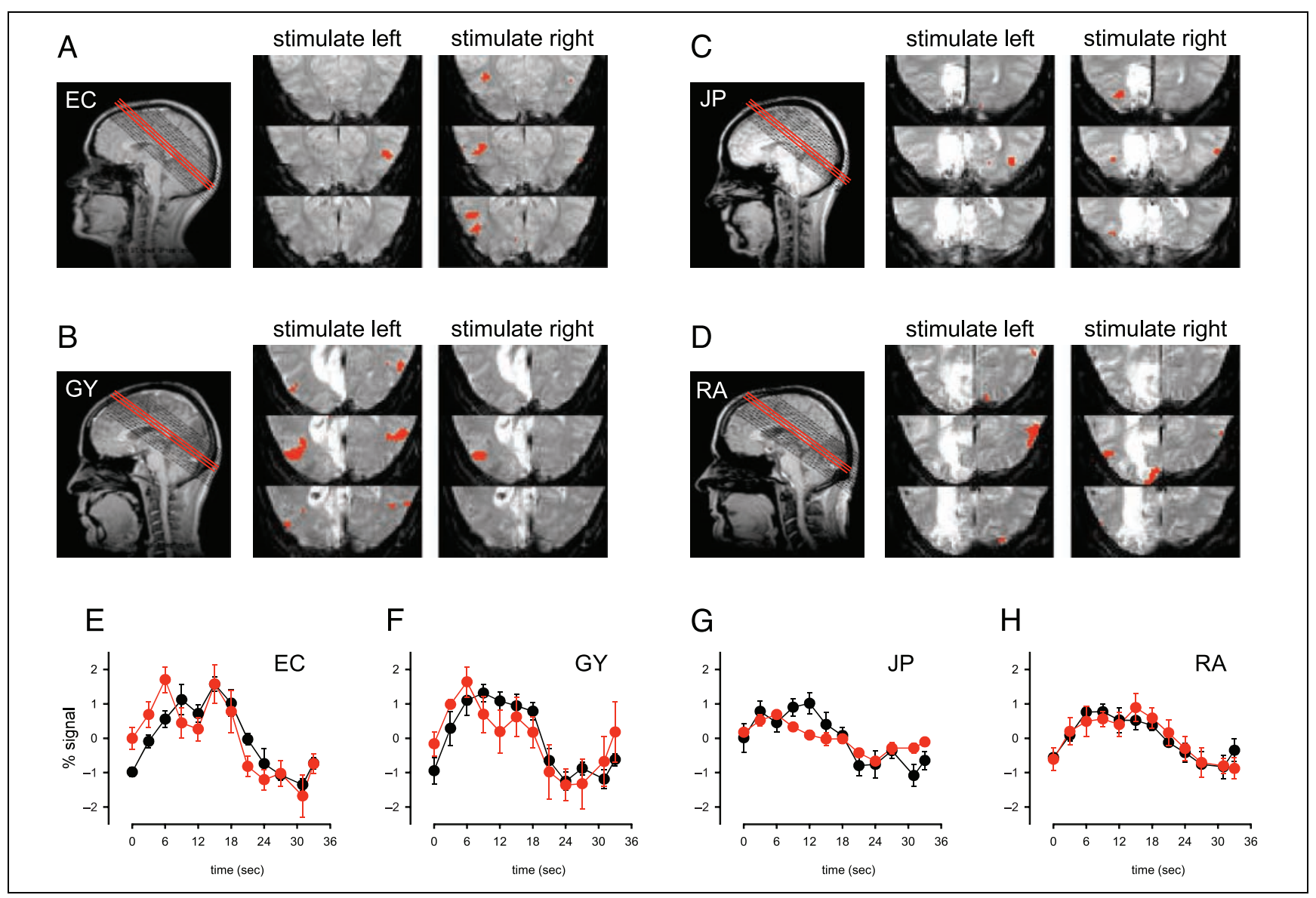

Figure 4. Cortical activity in response to stimulus motion. Voxels that exceed a correlation of .23 after spatial smoothing are represented in red on the mean $\mathrm{T} 2 *$ images. Images are presented in neurological convention. The slice positions are indicated in each panel (A-D) by red lines superimposed on the parasagittal scout image. In panels A-C subjects display robust lateral occipital activity but no evidence of extensive activity in the medial aspects of the occipital lobe. In D, however, the hemianope RA appears to have medial occipital activity when his affected field is stimulated. Panels E-H show the mean BOLD signal for voxels in the lateral occipital areas shown in the panels A-D. Data are given for stimulation of the left (in black) and right (in red) hemifields, where the right hemifield is the one in which all patients have their field defect.

result indicates that overall the sensitivity of the fMRI procedures appears equal for the intact pathways of the patients and controls. In order to examine further the cortical response, the time series (TS) from the active voxels from each hemisphere of the control subjects and the intact hemispheres of the patients are given in Figure 3. The mean TS of each group accurately reflects the temporal signature of the blood oxygenation level dependent (BOLD) response as previously measured by others in visual areas of the brain (e.g., Boynton, Engel, Glover, \& Heeger, 1996). The signal amplitudes vary between $0.5 \%$ and $2 \%$ in both the controls and patients. This value of signal modulation is consistent with other studies of visual responses to high contrast stimuli with fMRI (Boynton et al., 1996). The data in Figure 3 underline, therefore, that the fMRI technique is equally sensitive in the normal and patient groups.

The results described above confirm that the visual stimulation and fMRI acquisition paradigm were successful in revealing cortical activity in the lateral occipital areas in the normal visual pathway. One of our main goals, however, was to determine what cortical activity would be elicited by presenting moving stimuli to the lesioned visual system. For hemianopes AA, SB, RL, RC, and $\mathrm{PF}$, no activations exceeding a correlation of .23 were observed (Table 3) when the hemianopic field was stimulated. As all these patients were unable to discriminate motion, the lack of lateral occipital activity is consistent with the idea that it is necessary for motion discrimination in hemianopes. In the hemianopes GY, RA, and JP, cortical activation was observed in lateral occipital areas during stimulation of their scotomata (Table 3). In these cases, therefore, we provide positive evidence that activity in the lateral occipital areas is correlated with the ability to discriminate motion.

To examine the locus of the cortical activation in response to stimulus motion, active voxels have been superimposed on mean T2* images in Figure 4. Moving stimuli elicited cortical activity in the control, EC, in lateral occipital regions, with very little medial occipital 
activity in either hemisphere in either condition (Figure 4A). In GY, stimulation of the intact hemifield with moving stimuli elicited bilateral activity in the lateral occipital areas, with no activity observed in the medial occipital areas (Figure 4B). Stimulation of GY's scotoma resulted in lateral occipital activity in only the hemisphere contralateral to the visual stimulation (Figure $4 \mathrm{~B}$ ). There was, however, no evidence of activity in the medial occipital regions. Lateral occipital activation contralateral to the visual stimulation in both hemifields was also observed in JP (Figure 4C) and RA (Figure 4D). In $\mathrm{JP}$, the activity resulting from stimulating the right hemifield is likely to be a result of the stimulus falling on intact regions of the visual field (see Figure 2E). In RA, there is evidence of activation in medial aspects of the occipital lobe in the damaged hemisphere when the right hemifield was stimulated. The calcarine cortex could therefore provide input to the activated lateral occipital areas. In contrast to the patient JP, however, RA had no visual sensitivity when tested with perimetry, so the medial occipital activity that we documented is surprising. The TS of the BOLD signal in response to stimulation of the intact and hemianopic fields are also plotted in Figure 4E-H for each of the patients and the control. It is clear that the signals derived from the lateral aspects of the occipital lobe from each hemisphere are essentially equal in the control, EC, and the patients, GY and RA. In JP, however, the signal derived from the damaged hemisphere has smaller amplitude than that from the intact hemisphere. In this case, the difference in signal strength may originate from the very small number of voxels that contribute to the time-series data for JP.

It should be noted that the activation in all other controls followed a very similar pattern, with predominant lateral occipital activity but with few or no active voxels in the medial occipital areas. The loci of these activations along with those of the hemianopes are compared in Figure 5, where the center of the mass of the lateral occipital activations contralateral to stimulation is plotted for each subject. The loci are very similarly located and no systematic difference in location is observed between patients and controls. In fact, the spread of the centroids of the loci is not dissimilar from the extent of the activity we observed in individual subjects (Figure 4).

The second aim of our imaging experiments was to determine whether the calcarine cortex might be a

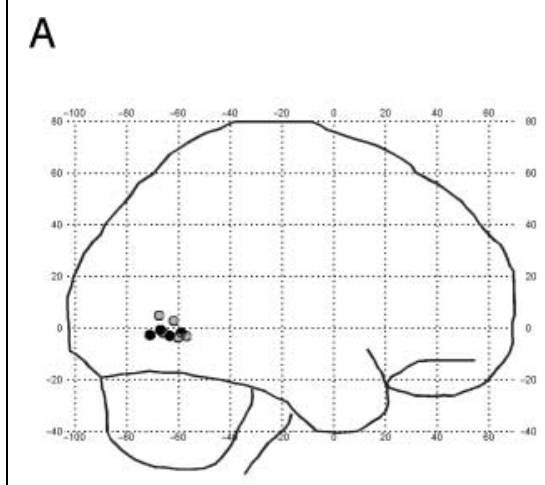

\section{left hemifield stimulation}
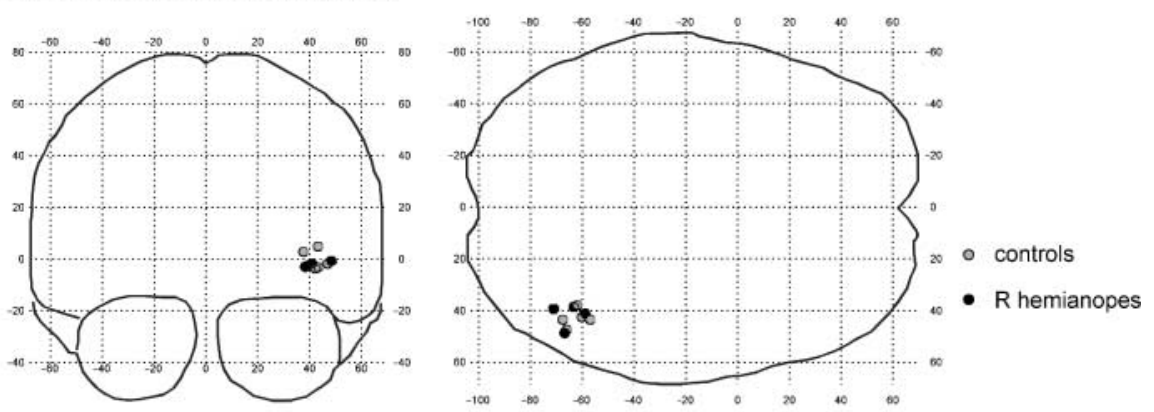

B
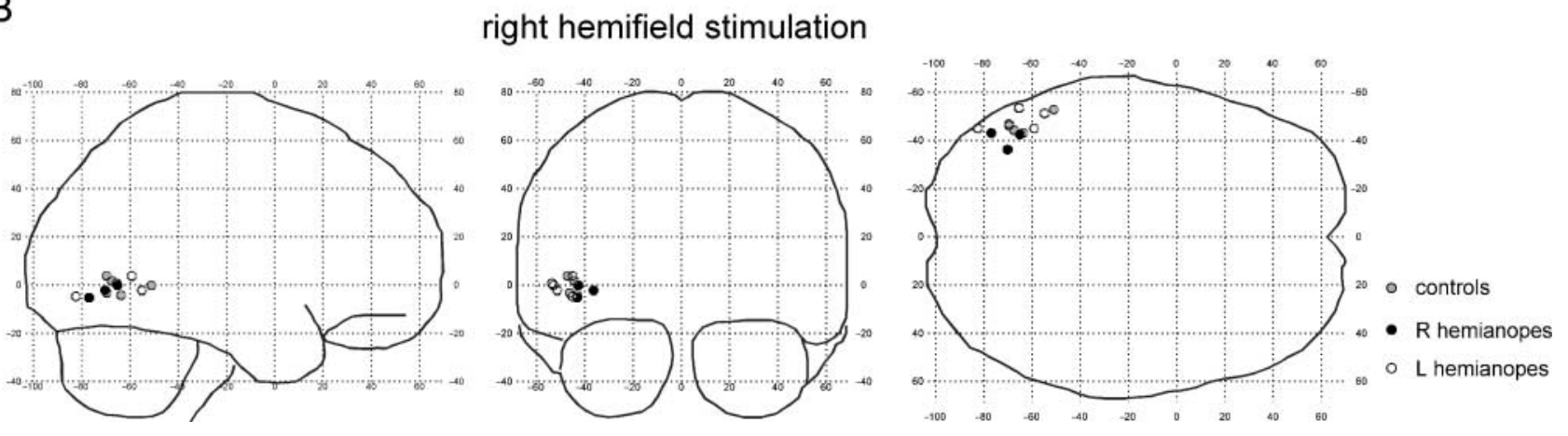

Figure 5. Coordinates of the cortical activations found in the lateral occipital regions in response to stimulation with moving gratings contrasted with static gratings. (A) Subjects were stimulated in the left hemifield. Note that only control subjects (gray circles) and hemianopic patients with intact left hemifields (black circles) displayed cortical activations for this condition. (B) Stimuli were presented in the right hemifield. In this condition, all hemianopes with intact right hemifields (open circles) and controls (gray circles) displayed activity, and three of the four hemianopes with scotomata in the right hemifield showed activations. The loci of activations were calculated as the center of mass of the contiguous regions of active voxels for each subject and were based on the normalized coordinate scheme of Talairach and Tournoux (1988). 


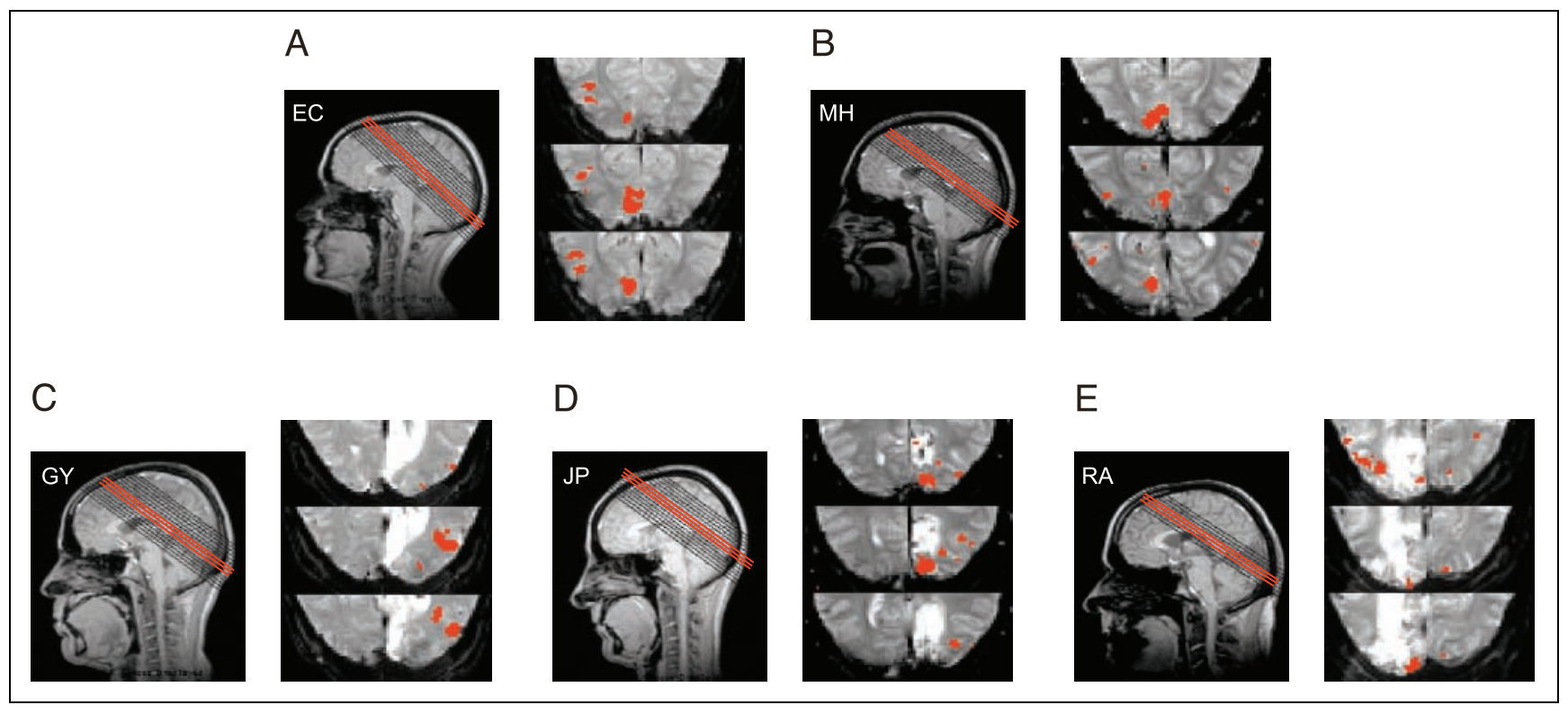

Figure 6. Cortical activity in response to counterphase grating stimulation. Distribution of active voxels that exceeded a correlation of .23 are plotted in red on the mean T2* images for two controls (A and B) and three hemianopes (C-D). Images are presented in neurological convention. The slice positions are indicated by the red lines in the parasagittal scout images for each subject. Note that the stimulation reliably elicited activity in both the medial and lateral aspects of the occipital lobe in normal controls. The medial activity was absent in GY (C) but was present in JP (D) and RA (E).

source of input to the lateral occipital areas and thereby mediate motion discrimination. A further experiment was necessary to examine this issue, because moving stimuli contrasted with static stimuli failed to elicit reliably cortical activity from the medial aspects of the occipital lobe in the normal visual system (both control and intact hemispheres of hemianopes; see Figure 4). Our second imaging experiment used a flickering grating stimulus contrasted with uniform gray. The resulting cortical activity was predominantly located in the medial aspects of the occipital lobe in the normal controls (Figure $6 \mathrm{~A}$ and $\mathrm{B}$ ) and the intact hemispheres of the patients (not illustrated). In addition to the medial occipital activity, lateral occipital activity was also seen in the control hemispheres (e.g., Figure 6A) in very similar locations to the activity elicited by motion (e.g., Figure 4A). In the damaged hemispheres of the three patients, two patterns of activity emerged: In GY, activity was observed only in the lateral occipital areas; in RA and JP, activity was observed in the medial occipital cortex, with further lateral occipital activity. In GY, therefore, activity in the lateral occipital areas can be elicited in the absence of any measurable activity in the calcarine cortex. In JP, we obtained an expected result: Stimulation of an intact region of the visual field, albeit in the scotomatous hemifield, results in activity of both medial and lateral occipital areas as it does in controls and the intact hemispheres of patients. In RA, standard perimetry revealed no sensitivity in the right hemifield, but we were able to confirm our previous imaging result, where we detected activity in the medial aspect of the occipital lobe. In this patient, therefore, we have evidence for spared islands in the medial occipital areas and this preserved activity could contribute to visual sensitivity to the large stimuli used in our motion discrimination task. It should also be noted that in each subject the activity recorded was always contralateral to the stimulated field, which indicates that patients were not fixating such that the stimulus was brought into their intact hemifield.

Until now, our data analysis has primarily been focused on statistically excluding false positives, which can lead to making a false-negative error (type II error). To assess whether our raw unblurred data displays any small, localized activity in GY's calcarine cortex that could have been missed by our techniques, we have adopted the following procedure. Regions of interest were identified in the medial and lateral aspects of the occipital lobe to cover the intact cortex at potential loci of activations. Within the regions of interest, the number of voxels that exceeded the correlation threshold $c>.23$ was calculated. Regions of interest outside the brain were then selected and the number of voxels within those regions that exceeded the threshold $(c>$ .23) was calculated. This number was then normalized to assess the proportion of voxels that would be expected of an empirical estimate of chance. The proportion was then used to calculate the number of voxels within the lateral and medial occipital regions of interest that would be expected to be correlated by chance. Thus, two voxel counts were established for each region of interest: one count was the observed number of voxels, 


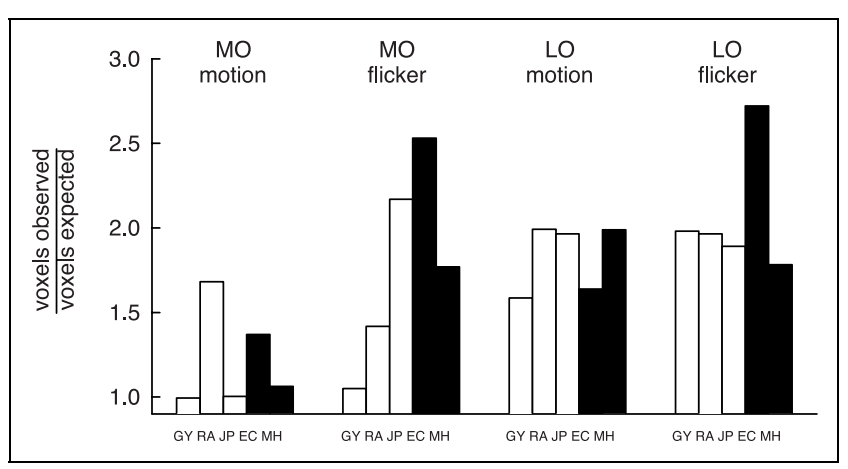

Figure 7. The ratio of observed active voxels to the number of active voxels expected by chance (at a correlation threshold of .23). Data are given for five subjects: three hemianopes (GY, RA, and JP) and two controls (EC and $\mathrm{MH}$ ). Ratios were calculated for experiments that contrasted motion with static gratings (motion) and flickering gratings with uniform gray (flicker). Data are given for the medial occipital (MO) and lateral occipital (LO) cortical regions.

and the other the number of voxels expected by chance. The ratio between the observed and the expected voxel counts is plotted for each subject and region of interest under motion and flicker conditions in Figure 7. The observed number of voxels only exceeded the expected number in the medial occipital area during motion stimulation for the hemianope RA and control EC. This indicated that the contrast between the moving and static stimuli was not consistent in differentially activating early visual areas. The experiment in which flicker was contrasted with uniform gray resulted in a larger number of observed than expected voxels in the medial occipital regions for the controls and hemianopes RA and JP. Importantly, however, the observed number of voxels in the GY's medial occipital area did not exceed the number of voxels expected by chance. This analysis, therefore, is in agreement with the absence of voxels reported in the data analysis that involved blurring the voxels (Figures 4 and 6). The lateral occipital regions in all subjects and for both experiments exhibited larger number of observed than expected voxels. The observed and expected voxel counts lend themselves to analysis with the $\chi^{2}$ test, which has been applied to the data shown in Figure 7. The $\chi^{2}$ values and their associated probability values are reported in Table 4 . It is clear that this analysis reveals that the number of voxels observed in GY's medial occipital cortex are no different than the number expected by chance with probabilities approaching unity (Table 4).

To assess the location of the medial occipital activation in JP and RA more closely, we compare the position of active regions in these patients' damaged hemispheres with those of intact hemispheres of the patients and control in Figure 8 . We generated a coordinate frame in which the location of activity could be compared by taking the following steps. (1) The $z$-dimension is defined as the direction perpendicular to the plane in which data were acquired with an origin that is defined by the position of the calcarine sulcus. (2) The $x$ dimension measures distance within the plane of acquisition from the vertical division of the occipital lobes. (3) The $y$-dimension measures the distance from the most posterior boundary of the cortex within the acquisition plane. The most important aspect of this registration scheme is the definition of the $x$-dimension with an origin at the division of the occipital lobes. Some of the patients' lesions distort the midline, so the scheme we have adopted is more appropriate than an affine transformation of the brain whole volume to a single template that would essentially preserve the asymmetry of the occipital lobes in the patients. We also believe that

Table 4. Results of a Chi-Square Analysis of the Observed and Expected Number of Voxels Counted in the Medial and Lateral Occipital Cortex

\begin{tabular}{|c|c|c|c|c|c|}
\hline \multirow[b]{2}{*}{ Subject } & \multirow[b]{2}{*}{ Experiment } & \multicolumn{2}{|c|}{ Medial Occipital } & \multicolumn{2}{|c|}{ Lateral Occipital } \\
\hline & & $x^{2}$ & $p$ & $x^{2}$ & $p$ \\
\hline \multirow[t]{2}{*}{ GY } & "flicker" & 0.0603 & 0.81 & 17.9 & $2.3 \times 10^{-5}$ \\
\hline & "motion" & $3.35 \times 10^{-3}$ & 0.95 & 82.3 & $7.2 \times 10^{-20}$ \\
\hline \multirow[t]{2}{*}{ RA } & "flicker" & 10.4 & $1.2 \times 10^{-3}$ & 52.0 & $5.4 \times 10^{-13}$ \\
\hline & "motion" & 25.5 & $4.3 \times 10^{-7}$ & 17.5 & $2.9 \times 10^{-5}$ \\
\hline \multirow[t]{2}{*}{ JP } & "flicker" & 80.6 & $2.8 \times 10^{-19}$ & 74.3 & $6.8 \times 10^{-18}$ \\
\hline & "motion" & $6.48 \times 10^{-4}$ & 0.98 & 10.1 & $1.5 \times 10^{-3}$ \\
\hline \multirow[t]{2}{*}{$\mathrm{EC}$} & "flicker" & 159 & $1.7 \times 10^{-36}$ & 152 & $5.2 \times 10^{-35}$ \\
\hline & "motion" & 9.31 & $2.3 \times 10^{-3}$ & 29.7 & $5.0 \times 10^{-8}$ \\
\hline \multirow[t]{2}{*}{ MH } & "flicker" & 11.2 & $8.2 \times 10^{-4}$ & 15.1 & $1.0 \times 10^{-4}$ \\
\hline & "motion" & 0.074 & 0.79 & 15.8 & $6.9 \times 10^{-5}$ \\
\hline
\end{tabular}




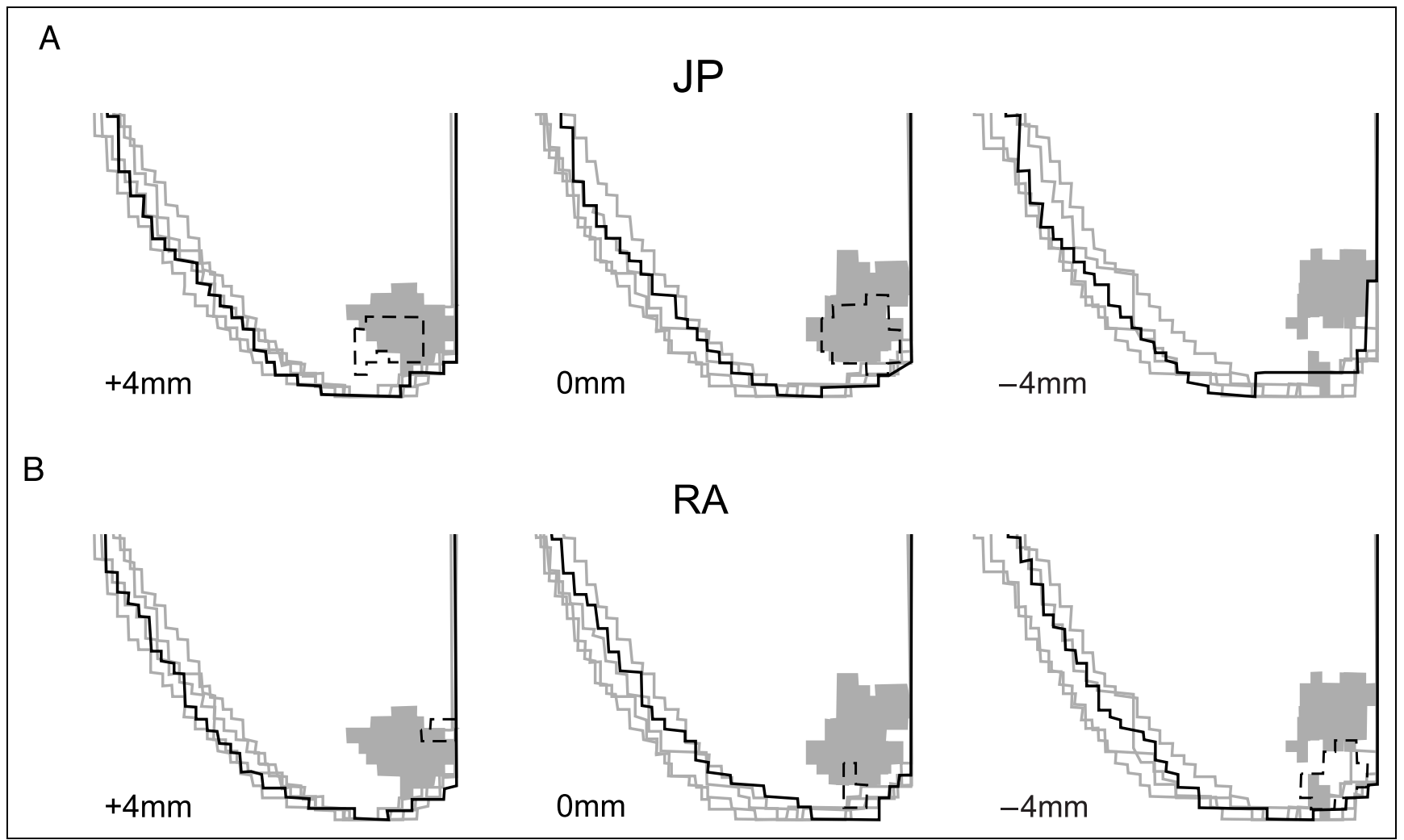

Figure 8. Medial occipital activity for JP and RA are compared with a template of normal cortical activity of the medial occipital lobe in A and B, respectively. The locus of JP's activity shown in the broken line in A overlaps greatly with the template locus (gray) in the calcarine sulcus ( $0 \mathrm{~mm}$ ) and $4 \mathrm{~mm}$ above it. For RA, there is less overlap of his medial occipital activity (broken line) with that of the template (gray). There is also less extensive contiguous activity in this subject. See text for details of how the template was established.

our identification of the calcarine sulcus again provides a more accurate means of determining a common coordinate frame in which calcarine activity can be compared. Data for all subjects in Figure 6 were acquired from three slices that were aligned with the calcarine sulcus and $4 \mathrm{~mm}$ above and below it. The data for all subjects, therefore, are essentially registered within the $z$-dimension. All that remains to assess the areas of activation is to register the data with respect to the $x$-and $y$-dimensions. This registration is performed in Figure 8 in which four control hemispheres (one from each of the controls EC and $\mathrm{MH}$ and the intact hemispheres [that are flipped left to right] of GY and JP) are aligned to generate a template of calcarine activity for stimulation with the flickering grating. Note that only medial occipital activity that can be associated with the calcarine sulcus has been plotted in gray. In JP, stimulation of the affected hemifield results in activity that has considerable overlap with that found in the normals at $z=0$ and $4 \mathrm{~mm}$. This is expected on the basis of the stimulus falling on the intact region of the inferior visual field (Figure 2E). The absence of activity at $z=-4 \mathrm{~mm}$ is also consistent with a lack of visual sensitivity in the superior visual field (Figure 2E). In RA, however, the overlap with the collective control data and the extent of activity is small. The extent of the activity is consistent with a spared "islands" of cortex that could subserve residual sensitivity to a large stimulus, but that this visual sensitivity would go undetected by conventional perimetry. The locus of the activity appears to indicate that some visual sensitivity may remain in RA's upper right quadrant between eccentricities of $5^{\circ}$ and $7.5^{\circ}$. The size of this zone could escape detection with targets that are separated by $6^{\circ}$, as were used in the Humphrey perimetry. The visual field location of the spared island that we have extrapolated must be taken with considerable caution, however, because of the significant structural distortion evident in RA's damaged hemisphere (Figures $2 \mathrm{C}$ and $6 \mathrm{E}$ ). By using the anatomical landmark of the calcarine sulcus, the activity shown in Figures 6 and 8 is likely to reflect that in the primary visual cortex, V1, of all subjects. We have been able to confirm this in the controls EC and $\mathrm{MH}$ by performing retinotopic mapping experiments, but we did not undertake such experiments on the patients, so we can only infer that the activity is within V1 for the hemianopes.

\section{DISCUSSION}

Our main findings can be summarized as follows. (1) Hemianopes who, in addition to a homonymous visual 
defect, have lateral occipital lesions are unable to discriminate motion direction and do not have any measurable cortical activity when stimulated with moving stimuli within their field defects. (2) Some hemianopes with medial occipital lesions and sparing of lateral occipital areas are able to discriminate motion direction. (3) In those hemianopes who were able to discriminate motion direction (GY and RA), activity in the lateral occipital areas was observed on stimulation of the field defect with drifting gratings. (4) We established that in one hemianope (RA) the lateral occipital activity could arise from input from "islands" of the intact calcarine cortex, whereas in the other hemianope (GY) this was not the case.

The absence of discrimination of motion direction in hemianopes with lateral occipital lesions confirms a previous study by Barton and Sharpe (1997), who used random dot cinematograms to determine direction discrimination in patients with lateral occipital lesions. Our fMRI results also revealed that there was no motion-specific activity in any cortical area when these patients' scotomata were stimulated with drifting gratings. It appears likely, therefore, that the lateral occipital areas that have been shown to respond to motion in normal subjects (Huk, Ress, \& Heeger, 2001; Huk \& Heeger, 2002; Culham et al., 1999; Barton et al., 1996; Tootell \& Taylor, 1995; Tootell et al., 1995a, 1995b; Zeki, 1991; Zeki et al., 1991) are required by patients in order for them to perform discriminations of motion direction.

We were able to document motion direction discrimination in two patients with lesions of the medial occipital areas. These behavioral responses are generally consistent with previous studies of motion discrimination on GY using a variety of moving stimuli (Morland et al., 1999; Benson et al., 1998; Zeki \& Ffytche, 1998; Finlay et al., 1997; Sahraie et al., 1997; Weiskrantz et al., 1995; Barbur et al., 1980, 1993) and other patients who have lesions that principally involve the geniculostriate projection (Perenin, 1991; Blythe et al., 1986). It should be noted, however, that one study has failed to show that GY and another hemianope could discriminate the direction of motion of drifting gratings (Azzopardi \& Cowey, 2001).

We found that extrastriate activity in the lateral occipital areas was present in the hemianopes who could discriminate motion direction. The strength of the signals we recorded was the same in the damaged and intact hemispheres of GY and RA. Our data are entirely consistent with previous imaging experiments that revealed lateral occipital activity in GY (Goebel et al., 2001; Zeki \& Ffytche, 1998; Baseler et al., 1997; Barbur et al., 1993) and another frequently studied patient, FS (Goebel et al., 2001; Stoerig et al., 1998). The experiment we performed, however, cannot determine whether the neural substrate remains directionally selective, a result that has only been demonstrated in normal subjects (Huk et al., 2001). In common with previous studies on motion-specific activity (Culham et al., 1999; Smith, Greenlee, Singh, Kraemer, \& Hennig, 1998; Barton et al., 1996; Tootell \& Taylor, 1995; Tootell et al., 1995a; 1995b; Zeki, 1991; Zeki et al., 1991), the stimulus contrast that we compared elicited little or no activity in the calcarine visual cortex in normal subjects. This single imaging experiment is not capable, therefore, of determining the extent to which signals in the calcarine cortex may contribute to the extrastriate motion-specific activity. It is noteworthy, however, that in one hemianope, RA, who discriminated motion direction, we found calcarine activity in response to the motion stimulus: A preliminary indication that spared calcarine cortex could mediate residual visual responses.

fMRI has been used previously to determine whether there are spared islands of activity within early visual areas of hemianopes, who can perform visual discriminations in their scotomata (Goebel et al., 2001; Stoerig et al., 1998). The results of those studies were negative for the subjects tested (GY and FS), so in these cases it appears that extrastriate activity, presumably innervated through subcortical routes, is sufficient to mediate their residual visual function (Goebel et al., 2001; Stoerig et al., 1998). In this study, we confirm the previous observations on GY: We found that when we presented a stimulus, which elicited robust activity in the calcarine cortex of his intact hemisphere and the control hemispheres, no measurable activity could be found in the remaining calcarine cortex of the damaged hemisphere. The absence of measurable calcarine activity does not rule out completely the presence of small islands of functioning neurons that fail to elicit a BOLD response because the islands are too small to drive a measurable hemodynamic response or because the neurovascular coupling may be disrupted in the lesioned area. There are, however, two features that make the presence of such islands unlikely candidates for providing GY with the ability to discriminate motion direction for the stimuli we used here. Firstly, GY has not been shown to exhibit any spared islands of behavioral sensitivity in his scotomatous field (Kentridge et al., 1997). Secondly, the extent of GY's calcarine lesion includes all the tissue that is normally devoted to the representation of the stimulus used here.

In the other hemianope with residual vision, RA, we found a different pattern of results to those documented in GY. Our experiments revealed activity in small regions of spared calcarine cortex, that were located in very similar positions to the locations of activity in the calcarine cortex in response to motion. This result at first appears similar to those of Celesia et al. (1991), although these authors studied patients with clearly identifiable intact regions of conscious vision, characteristics that are more similar to our patient JP. For the first time, therefore, we provide positive 
evidence that small regions of the calcarine cortex can be spared and remain responsive to visual stimulation in a patient (RA) who is cortically blind as defined by standard perimetry. It should be noted, however, that the experiments we undertook are not able to resolve whether the residual activity we document in V1 provides the input to the lateral occipital areas involved in motion processing. Further imaging experiments that allow analysis of functional connectivity between V1 and motion areas may be able to shed light on this issue. It remains clear, however, that our overall results are consistent with two pathways that mediate human residual visual function: one that bypasses V1, and the other that relies on small intact and active regions of $\mathrm{V} 1$.

Research on some hemianopes can reveal the capacities of the visual pathways that circumvent the primary visual cortex. In other hemianopes, however, it appears that residual capacities may be mediated by spared islands of V1. It is essential, therefore, to document with sophisticated perimetry measurements, functional imaging, or preferably both, the presence of any spared regions of sensitivity in the visual field in human hemianopes before assigning functional significance to the residual visual capacities that may be displayed by an individual patient.

\section{METHODS}

\section{Behavioral Measurements}

Visual fields were assessed with the Humphrey Visual Field Analyser with the 30-2 threshold routine. This routine presents $0.5^{\circ}$ static targets for duration of $0.2 \mathrm{sec}$ over the central $\pm 30^{\circ}$. Subjects view the targets at a distance of $33 \mathrm{~cm}$ and wear reading spectacles if necessary. Targets are located in a fixed grid of positions with separations of $6^{\circ}$. The thresholding routine varies the target luminance to establish the limit of target visibility at each location. In the patients we tested highest target luminance (10,000 astib) remained undetected in many field locations. These locations are denoted by filled squares in the visual field plots presented here.

We measured motion direction discrimination in all subjects with drifting grating stimuli. The sinusoidal grating $\left(10^{\circ} \times 10^{\circ}\right.$, spatial frequency: 0.5 or 1 cycle/ deg, contrast: $90 \%$, mean luminance: $80 \mathrm{~cd} \mathrm{~m}^{-2}$ ) was presented for 3 sec and drifted either leftward or rightward. Subjects viewed a stationary fixation cross, which was $5^{\circ}$ from the nearest side of the drifting grating, and were presented eight blocks of 40 trials in which the direction of motion was varied pseudorandomly. For two of the blocks the grating drifted at $10^{\circ} \mathrm{sec}^{-1}$ and for the other two the grating speed was $5^{\circ} \mathrm{sec}^{-1}$. Each hemifield was tested with both speeds and both spatial frequencies; thus eight different stimulus conditions were tested. The gratings were gener- ated on an LCD monitor (NEC 2010), which was also used to present stimuli in the MR scanner. The area of the screen surrounding the grating was set to the maximum screen luminance $\left(180 \mathrm{~cd} \mathrm{~m}^{-2}\right)$ to reduce any cues that could be derived from scattered light. The subject's task was to indicate whether the grating motion was to the right or to the left. Trials were self-paced, but the experimenter recorded the data with keyboard presses following a verbal response from the subject.

\section{MRI Measurements}

Anatomical images were obtained using a Siemens Magnetom Vision 1.5-T MRI system using the MPRAGE sequence with a voxel size of $0.98 \times 0.98 \times 1 \mathrm{~mm}$. The functional MR images were acquired using the same scanner, which was fitted with EPI gradient overdrive. A Multi-slice 2-D gradient-echo EPI sequence (TE $54 \mathrm{msec}$, 128 by 128 matrix, $240 \mathrm{~mm}$ field of view, interleaved slice order with no gap) was used to measure the BOLD signal as a function of time. The image planes were $4 \mathrm{~mm}$ thick, giving voxel dimensions of $1.82 \times 1.82 \times$ $4 \mathrm{~mm}$. The functional images were acquired once every 3 sec during visual stimulation, which followed a block design. Data were acquired in either 8 or 14 slices at an orientation that was parallel to the calcarine sulcus. A mirror mounted on the head coil angled at $45^{\circ}$ with respect to the frontal plane allowed subjects to see along the bore of the scanner above their heads. At the end of the bore, another mirror angled at $-45^{\circ}$ with respect to the frontal plane allowed a screened LCD panel mounted on the scanner room floor to be viewed.

In designing our visual stimulation procedures for fMRI, we wanted to (1) reproduce as closely as possible visual stimulus conditions used to obtain behavioral responses and (2) follow a block design that elicits activity most reliably. In meeting these requirements, we settled on presenting stimulus trials of the same length as used in the behavioral tests and grouping these trials in blocks so as to generate a suitable contrast in conditions. We also required two contrast conditions: one that was motion specific and the other that would activate the calcarine cortex.

Our initial design of a motion-specific stimulus contrast involved blocks of four trials of a drifting grating with control blocks comprising four trials of the same grating undergoing counterphase flicker at the same frequency. This was unsuccessful because we could not reliably identify cortical activations in normal controls. We were more successful when the control block comprised four trials of the static grating. The stimulus design is summarized as follows. In the "motion" block we presented four trials in which a grating ( 1 cycle/deg) randomly drifted (at $5 \mathrm{~Hz}$ ) to the right or left. In the "static" block four trials were presented in which the 
grating was now static, but in every other respect identical to the grating presented in the "motion" trials. The four trials in the "motion" and "static" blocks were each of $3 \mathrm{sec}$ duration and were separated by $1.5 \mathrm{sec}$. A "static" block followed each "motion" block such that a stimulus cycle was $36 \mathrm{sec}$ in duration.

Although the experimental design described above was successful in eliciting activity in the lateral occipital areas in normals, it seldom elicited activity from the calcarine cortex. Because we wished to determine whether islands of spared tissue in the primary visual cortex were involved in visual responses of hemianopes, we required another stimulus design to reliably elicit activity within that visual area in normals. We found that a block of four trials of the grating undergoing counterphase flicker at $5 \mathrm{~Hz}$, followed by a control block in which a uniform gray was presented, elicited activity in V1 in normal control subjects. In the "flicker" block the four trials were identical and were each of $3 \mathrm{sec}$ duration and separated by $1.5 \mathrm{sec}$. The "gray" block lasted $18 \mathrm{sec}$, so a single stimulus cycle was $36 \mathrm{sec}$ in duration.

For all experiments, the stimulus cycles were repeated seven times such that each experiment lasted $252 \mathrm{sec}$. We repeated the experiments twice in the two stimulus locations left and right of fixation. In all but one case (RC), this allowed us to average the TS. RC moved too much during scans for data to be used in some experimental runs so only one TS could be assessed. Data were corrected for motion before analysis.

To assess cortical activity, each voxel's TS underwent following analysis: (1) The first cycle of stimulation (12 temporal samples) was discarded from analysis to avoid transient onset artifacts associated with magnetization not reaching a steady state; (2) The linear trend over the 84 temporal samples was removed; (3) The TS was divided by the voxel's mean intensity; (4) Fourier analysis was applied to obtain the amplitude and phase for each frequency; (5) The correlation with respect to the fundamental frequency of the visual stimulation, $1 / 36 \mathrm{~Hz}$, was calculated. The voxels for which correlation was less than .23, were then discarded. The threshold value of .23 was preselected before the study as it represents the correlation at which $p=.05$ for 72 temporal samples. The voxel map of "active" voxels was then spatially blurred, which serves the purpose of smoothing contiguous regions of activation. Blurring also has the effect of removing isolated single voxels that exceed the correlation threshold most probably due to chance. The spatial blurring, therefore, removes false-positive data just as an application of the Bonferroni correction for multiple comparisons would do. In addition, we also performed analysis on the unblurred data to evaluate whether blurring had removed significant activity and thereby cause a type II error (see Results section).

Our experimental procedures were applied to the sighted and hemianopic field in each subject: Each subject acted as their own control. In addition to this internal control, we also applied our experiments to four control subjects, who had no known visual deficits. All hemianopic subjects were recruited from an established cohort. None of them were hospitalized at the time of testing and had suffered their cortical lesions at least 4 years before undergoing our experiments. All subjects gave informed consent and the study was approved by the Riverside Ethics Committee.

\section{Acknowledgments}

We thank the Wellcome Trust (04066) for the financial support provided for this study. We are also indebted to the participants for their willingness to take part in a lengthy series of experiments. Finally, we are grateful to the staff at the Radiological Sciences Unit, Charing Cross Hospital, without whom the study would not have been possible.

Reprint requests should be sent to Antony B. Morland, Psychology Department, Royal Holloway, University of London, Engham, Surrey, TW20 0EX, UK, or via e-mail: A.Morland@ rhul.ac.uk.

The data reported in this experiment have been deposited in the fMRI Data Center (http://www.fmridc.org). The accession number is 2-2003-1144D.

\section{REFERENCES}

Azzopardi, P., \& Cowey, A. (2001). Motion discrimination in cortically blind patients. Brain, 124, 30-46.

Barbur, J. L., Ruddock, K. H., \& Waterfield, V. A. (1980). Human visual responses in the absence of the geniculo-calcarine projection. Brain, 103, 905-928.

Barbur, J. L., Watson, J. D., Frackowiak, R. S., \& Zeki, S. (1993). Conscious visual perception without V1. Brain, 116, 1293-1302.

Barton, J. J., Simpson, T., Kiriakopoulos, E., Stewart, C., Crawley, A., Guthrie, B., Wood, M., \& Mikulis, D. (1996). Functional MRI of lateral occipitotemporal cortex during pursuit and motion perception. Annals of Neurology, 40, 387-398.

Barton, J. J. S., \& Sharpe, J. A. (1997). Motion direction discrimination in blind hemifields. Annals of Neurology, 41, 255-264.

Baseler, H. A., Morland, A. B., \& Wandell, B. A. (1999). Topographic organization of human visual areas in the absence of input from primary cortex. Journal of Neuroscience, 19, 2619-2627.

Baseler, H. A., Wandell, B. A., Morland, A. B., Jones, S. R., \& Ruddock, K. H. (1997). Activity in the visual cortex of a hemianope. Proceedings of the 1997 Optical Society of America meeting "Vision Science and Its Applications", 1, 226-229.

Benevento, L. A., \& Yoshida, K. (1981). The afferent and efferent organization of the lateral geniculo-prestriate pathways in the macaque monkey. Journal of Comparative Neurology, 203, 455-474.

Benson, P. J., Guo, K., \& Blakemore, C. (1998). Direction discrimination of moving gratings and plaids and coherence in dot displays without primary visual cortex (V1). European Journal of Neuroscience, 10, 3767-3772.

Blythe, I. M., Bromley, J. M., Kennard, C., \& Ruddock, K. H. (1986). Visual discrimination of target displacement remains 
after damage to the striate cortex in humans. Nature, 320, 619-621.

Blythe, I. M., Kennard, C., \& Ruddock, K. H. (1987). Residual vision in patients with retrogeniculate lesions of the visual pathways. Brain, 110, 887-905.

Boynton, G. M., Engel, S. A., Glover, G. H., \& Heeger, D. J. (1996). Linear systems analysis of functional magnetic resonance imaging in human V1. Journal of Neuroscience, 16, 4207-4221.

Celesia, G. G., Bushnell, D., Toleikis, S. C., \& Brigell, M. G. (1991). Cortical blindness and residual vision: Is the "second" visual system in humans capable of more than rudimentary visual perception? Neurology, 41, 862-869.

Collins, C. E., Lyon, D. C., \& Kaas, J. H. (2003). Responses of neurons in the middle temporal visual area after long-standing lesions of the primary visual cortex in adult new world monkeys. Journal of Neuroscience, 23, 2252-2264.

Cowey, A., \& Stoerig, P. (1989). Projection patterns of surviving neurons in the dorsal lateral geniculate nucleus following discrete lesions of striate cortex: Implications for residual vision. Experimental Brain Research, 75, 631-638.

Culham, J. C., Dukelow, S. P., Vilis, T., Hassard, F. A., Gati, J. S., Menon, R. S., \& Goodale, M. A. (1999). Recovery of fMRI activation in motion area MT following storage of the motion aftereffect. Journal of Neurophysiology, 81, 388-393.

Fendrich, R., Wessinger, C. M., \& Gazzaniga, M. S. (1992). Residual vision in a scotoma: Implications for blindsight. Science, 258, 1489-1491.

Fendrich, R., Wessinger, C. M., \& Gazzaniga, M. S. (2001). Speculations on the neural basis of islands of blindsight. Progress in Brain Research, 134, 353-366.

Finlay, A. L., Jones, S. R., Morland, A. B., Ogilvie, J. A., \& Ruddock, K. H. (1997). Movement in the normal visual hemifield induces a percept in the "blind" hemifield of a human hemianope. Proceedings of the Royal Society of London. B, Biological Sciences, 264, 267-275.

Fries, W. (1981). The projection from the lateral geniculate nucleus to the prestriate cortex of the macaque monkey. Proceedings of the Royal Society of London. B, Biological Sciences, 213, 73-86.

Girard, P., \& Bullier, J. (1989). Visual activity in area V2 during reversible inactivation of area 17 in the macaque monkey. Journal of Neurophysiology, 62, 1287-1302.

Girard, P., Salin, P. A., \& Bullier, J. (1991a). Visual activity in areas V3a and V3 during reversible inactivation of area V1 in the macaque monkey. Journal of Neurophysiology, 66, 1493-1503.

Girard, P., Salin, P. A., \& Bullier, J. (1991b). Visual activity in macaque area V4 depends on area 17 input. NeuroReport, 2, 81-84.

Girard, P., Salin, P. A., \& Bullier, J. (1992a). Response selectivity of neurons in area MT of the macaque monkey during reversible inactivation of area V1. Journal of Neurophysiology, 67, 1437-1446.

Girard, P., Salin, P. A., \& Bullier, J. (1992b). Response selectivity of neurons in area-Mt of the Macaque monkey during reversible inactivation of area-V1. Journal of

Neurophysiology, 67, 1437-1446.

Goebel, R., Muckli, L., Zanella, F. E., Singer, W., \& Stoerig, P. (2001). Sustained extrastriate cortical activation without visual awareness revealed by fMRI studies of hemianopic patients. Vision Research, 41, 1459-1474.

Huk, A. C., \& Heeger, D. J. (2002). Pattern-motion responses in human visual cortex. Nature Neuroscience, 5, 72-75.
Huk, A. C., Ress, D., \& Heeger, D. J. (2001). Neuronal basis of the motion aftereffect reconsidered. Neuron, 32, 161-172.

Kasten, E., Wuest, S., \& Sabel, B. A. (1998). Residual vision in transition zones in patients with cerebral blindness. Journal of Clinical and Experimental Neuropsychology, 20, 581-598.

Kentridge, R. W., Heywood, C. A., \& Weiskrantz, L. (1997). Residual vision in multiple retinal locations within a scotoma: Implications for blindsight. Journal of Cognitive Neuroscience, 9, 191-202.

Morland, A. B. (1999). Conscious and veridical motion perception in a human hemianope. Journal of Consciousness Studies, 6, 43-53.

Morland, A. B., Jones, S. R., Finlay, A. L., Deyzac, E., Le, S., \& Kemp, S. (1999). Visual perception of motion, luminance and colour in a human hemianope. Brain, 122, 1183-1198.

Pasik, T., \& Pasik, P. (1971). The visual world of monkeys deprived of striate cortex: Effective stimulus parameters and the importance of the accessory optic system. Vision Research, Suppl 3, 419-435.

Perenin, M. T. (1991). Discrimination of motion direction in perimetrically blind fields. NeuroReport, 2, 397-400.

Riddoch, G. (1917). Dissociation of visual perceptions due to occipital injuries, with especial reference to appreciation of movement. Brain, 40, 15-17.

Rodman, H. R., Gross, C. G., \& Albright, T. D. (1989). Afferent basis of visual response properties in area MT of the macaque: I. Effects of striate cortex removal. Journal of Neuroscience, 9, 2033-2050.

Sahraie, A., Weiskrantz, L., Barbur, J. L., Simmons, A., Williams, S. C., \& Brammer, M. J. (1997). Pattern of neuronal activity associated with conscious and unconscious processing of visual signals. Proceedings of the National Academy of Sciences, U.S.A., 94, 9406-9411.

Scharli, H., Harman, A. M., \& Hogben, J. H. (1999). Residual vision in a subject with damaged visual cortex. Journal of Cognitive Neuroscience, 11, 502-510.

Smith, A. T., Greenlee, M. W., Singh, K. D., Kraemer, F. M., \& Hennig, J. (1998). The processing of first- and second-order motion in human visual cortex assessed by functional magnetic resonance imaging (fMRI). Journal of Neuroscience, 18, 3816-3830.

Standage, G. P., \& Benevento, L. A. (1983). The organization of connections between the pulvinar and visual area MT in the macaque monkey. Brain Research, 262, 288-294.

Stoerig, P., \& Cowey, A. (1997). Blindsight in man and monkey. Brain, 120, 535-559.

Stoerig, P., Faubert, J., Ptito, M., Diaconu, V., \& Ptito, A. (1996). No blindsight following hemidecortication in human-subjects. NeuroReport, 7, 1990-1994.

Stoerig, P., Kleinschmidt, A., \& Frahm, J. (1998). No visual responses in denervated V1: High-resolution functional magnetic-resonance-imaging of a blindsight patient. NeuroReport, 9, 21-25.

Talairach, J., \& Tournoux, P. (1988). Co-planar stereotaxic atlas of the buman brain 3-d proportional system: An approach to cerebral imaging. Stutgart: Thieme.

Tootell, R. B., Reppas, J. B., Dale, A. M., Look, R. B., Sereno, M. I., Malach, R., Brady, T. J., \& Rosen, B. R. (1995a). Visual motion aftereffect in human cortical area MT revealed by functional magnetic resonance imaging [see comments]. Nature, 375, 139-141.

Tootell, R. B., Reppas, J. B., Kwong, K. K., Malach, R., Born, R. T., Brady, T. J., Rosen, B. R., \& Belliveau, J. W. (1995b). Functional analysis of human MT and related visual cortical areas using magnetic resonance imaging. Journal of Neuroscience, 15, 3215-3230. 
Tootell, R. B., \& Taylor, J. B. (1995). Anatomical evidence for MT and additional cortical visual areas in humans. Cerebral Cortex, 5, 39-55.

Weiskrantz, L. (1986). Blindsight: A case study amd implications. Oxford: Oxford University Press.

Weiskrantz, L., Barbur, J. L., \& Sahraie, A. (1995). Parameters affecting conscious versus unconscious visual discrimination with damage to the visual cortex (V1). Proceedings of the National Academy of Sciences, U.S.A., 92, 6122-6126.

Weiskrantz, L., Warrington, E. K., Sanders, M. D., \& Marshall, J. (1974). Visual capacity in hemianopic field following a restricted occipital ablation. Brain, 97, 709-728.

Wessinger, C. M., Fendrich, R., \& Gazzaniga, M. S. (1997). Islands of residual vision in hemianopic patients. Journal of Cognitive Neuroscience, 9, 203-221.
Wust, S., Kasten, E., \& Sabel, B. A. (2002). Blindsight after optic nerve injury indicates functionality of spared fibers. Journal of Cognitive Neuroscience, 14, 243-253.

Yukie, M., \& Iwai, E. (1981). Direct projection from the dorsal lateral geniculate nucleus to the prestriate cortex in macaque monkeys. Journal of Comparative Neurology, 201, 81-97.

Zeki, S. (1991). Cerebral akinetopsia (visual motion blindness). A review. Brain, 114, 811-824.

Zeki, S., \& Ffytche, D. H. (1998). The Riddoch-syndrome: Insights into the neurobiology of conscious vision. Brain 121, 25-45.

Zeki, S., Watson, J. D., Lueck, C. J., Friston, K. J., Kennard, C., \& Frackowiak, R. S. (1991). A direct demonstration of functional specialization in human visual cortex. Journal of Neuroscience, 11, 641-649. 